\title{
Chronic Stress Promotes the Progression of Pressure Overload-Induced Cardiac Dysfunction Through Inducing More Apoptosis and Fibrosis
}

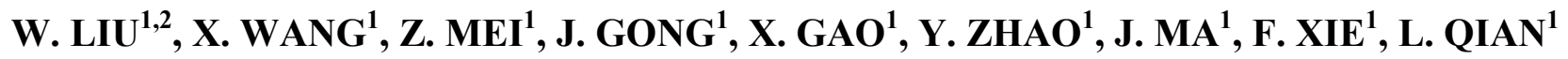 \\ ${ }^{1}$ Beijing Institute of Basic Medical Sciences, Beijing, China, ${ }^{2}$ Institute of Health and Environmental \\ Medicine, Tianjin, China
}

Received March 19, 2014

Accepted September 15, 2014

On-line December 22, 2014

\begin{abstract}
Summary
Stress serves as a risk factor in the etiology of hypertension. The present study was designed to decipher the effect and mechanism of chronic stress on the progression of pressure overload-induced cardiac dysfunction. We used abdominal aortic constriction (AAC) to induce pressure overload with or without chronic restraint stress to establish the animal models. Echocardiographic analysis showed pressure overload-induced cardiac dysfunction was worsened by chronic stress. Compared with the AAC rats, there is a significant increase in cardiac hypertrophy, injury, apoptosis and fibrosis of the AAC + stress rats. Furthermore, we found the secretion of norepinephrine (NE) increased after the AAC operation, while the level of NE was higher in the AAC + stress group. Cardiomyocytes and cardiac fibroblasts isolated from neonatal rats were cultured and separately treated with $1,10,100 \mu \mathrm{M}$ NE. The higher concentration NE induced more cardiomyocytes hypertrophy and apoptosis, cardiac fibroblasts proliferation and collagen expression. These results revealed that high level of NE-induced cardiomyocytes hypertrophy and apoptosis, cardiac fibroblasts proliferation and collagen expression further contributes to the effect of chronic stress on acceleration of pressure overloadinduced cardiac dysfunction.
\end{abstract}

\section{Key words}

Chronic stress - Pressure overload • Cardiac dysfunction • Norepinephrine

\section{Corresponding author}

L. Qian, Beijing Institute of Basic Medical Sciences, No. 27 Taiping Road, Beijing 100850, China. Fax: +86-10-68213039. E-mail: newjia@vip.sina.com

\section{Introduction}

Hypertension is one of the most common causes of cardiac remodeling and dysfunction, which eventually leads to heart failure (Cumbermack et al. 2011, Li et al. 2013, Manolis et al. 2014, Ribeiro et al. 2014). Heart failure is a final stage of most cardiovascular disease, which poses serious threats to human health and life (Levy et al. 2002). The progression of cardiac remodeling adversely promotes myocardial stiffness and left ventricular dilation, resulting in accelerating heart failure (Brilla et al. 1990). Cardiac remodeling is the convergent phenotype of various diseases that causes dysfunction of cardiac myocytes and cardiac fibroblasts (Kremneva and Abaturova 2003, Brown et al. 2005). Thus, preventing the progression of pressure overload is expected to suppress heart failure. A deeper understanding of the risk factors affecting the cardiac dysfunction in response to hemodynamic overload is essential to the development of effective preventative measures and therapies.

Stress is defined as the effects of environmental or psychosocial factors on physical or mental well-being. Stress overload can cause injury and even diseases, such as diabetes, gastric ulcer, obesity, cancer, and Parkinson's disease. Data suggest a relationship between stress and the risk of cardiovascular disease (Zhao et al. 2007). It 
has been found that the cardiovascular system is the major target of stress injury (Wang et al. 2012, Ippoliti et al. 2013). Stress has also been shown to be important to vascular hypertension (Spruill 2010, Yu et al. 2013), and it may serve as a risk factor, induce blood pressure spikes, or increase an already elevated blood pressure (Wenneberg et al. 1997, Lutgendorf et al. 2000). Nevertheless, it remains to determine whether chronic stress participates in the progression of pressure overloadinduced cardiac dysfunction. Therefore, the present study was undertaken to investigate the effect of chronic stress on the progression of the pressure overload-induced cardiac dysfunction, and explore the possible mechanism of the effects of chronic stress during the progression of pressure overload-induced cardiac remodeling.

\section{Materials and Methods}

\section{Experimental animals}

The male Wistar rats weighing 180-200 g were randomly divided into the following groups: sham operation, abdominal aortal constriction (AAC) operation and $\mathrm{AAC}+$ stress. The operation group was subjected to abdominal aortal constriction (AAC) with a 7-gauge syringe needle under anesthesia with sodium pentobarbital, the sham operation group was treated as previously described, and the AAC + stress group were subjected to chronic restraint stress for $6 \mathrm{~h}$ per day after 1 week of the AAC operation. The rats were sacrificed 2, 3 and 4 weeks after the operations under anesthesia. All animal experimental procedures were conducted in accordance with the Guide for Care and Use of Laboratory Animals (NIH Publication No. 85-23, revised 1996). The protocol was approved by the committee on the Ethics of Animal Experiments of the Beijing Institute of Basic Medical Sciences (Permit Number: 2012-D3096).

\section{Echocardiography and blood pressure analysis}

The rats were anesthetized by isoflurane and $\mathrm{O}_{2}$ inhalation. Blood pressure measurements were conducted using carotid artery cannulation with a MP150 polygraph (BIOPAC, USA). Echocardiographic measurements were conducted with a high-resolution echocardiography analysis system for small animals (Vevo770, Visual Sonics, Canada). A 2-dimensional short-axis view and M-mode tracings of the left ventricle (LV) were obtained with a 17.5-MHz RMV-716 transducer.
Histology

The left ventricle was separated, washed and fixed in $4 \%$ paraformaldehyde at $4{ }^{\circ} \mathrm{C}$ overnight, embedded in paraffin and sectioned at $5 \mu \mathrm{m}$. The sections were stained with hematoxylin/eosin to detect myocardial hypertrophy and with Masson's trichrome staining to assess fibrosis.

Measurement the contents of Serum levels of CK-MB and $T n I$

Enzyme linked immunosorbent assay (ELISA) kits were used to determinate the contents of creatine kinase isoenzyme (CK-MB) and troponin I (TnI) in rats serum. All assays were performed according to the manufacture's instruction.

\section{Caspase-3 activity assay}

Caspase- 3 activity in rat heart tissue was detected using the caspase-3 colorimetric activity assay kit (Genmed Scientific Inc., China). The assay was based on spectophotometric detection of the pNA after cleavage from the labeled substrate Ac-DEVD-pNA and performed according to the manufacture's instruction (Jin et al. 2013).

\section{Cell culture}

Cardiac myocytes and cardiac fibroblasts were isolated from the left ventricles of 1-2-day-old Wistar rats as previously described (Tsuruda et al. 1999). Cardiomyocytes with more than 100 times of spontaneous beating were used for the experiment. The identities of the CFs were confirmed by immunostaining for DDR2. Passages 2-4 were used for the experiments.

\section{Western blot analysis}

The heart tissue and cells were lysed and subjected to SDS-PAGE and transferred to PVDF membranes. Western blot analyses were conducted with commercially available antibodies (anti-BNP: Santa Cruz; anti-collagen I and collagen III: abcam). They were visualized with the use of SuperSignal® West Femto Maximum Sensitivity Substrate (Thermo Scientific, USA) and images were captured with an ImageQuant LAS 4000 (GE, USA).

\section{MTT assay}

Cell viability was determined using the MTT assay (Sigma-Aldrich, St. Louis, MO, USA). The cells were seeded onto a 96-well plate overnight. Then, cells 
were treated with the processing mode. After treatment, the medium was removed and $200 \mu \mathrm{l}$ of the MTT medium $(0.5 \mathrm{mg} / \mathrm{ml}$ MTT reagent in fresh medium) was added to each well, and the cells were incubated at $5 \%$ $\mathrm{CO}_{2}$ and $37^{\circ} \mathrm{C}$ for $4 \mathrm{~h}$. Finally, the MTT reagent was removed and $150 \mu \mathrm{l}$ DMSO was added to each well following $10 \mathrm{~min}$ of gentle shaking. The absorbance was measured at $490 \mathrm{~nm}$ with a microplate reader. All experiments were performed in eight wells and repeated three times.

\section{Flow cytometry}

For the apoptosis assays, annexin $\mathrm{V}$ staining was performed using the Annexin V-FITC Apoptosis Detection Kit (BD Biosciences, CA, USA) according to the manufacturer's recommendations. The samples were examined by flow cytometry (FACS Calibur, BD, USA), and the data were analyzed with CELLQuest software (FACS Calibur, BD, USA).

For the cell cycle assays, following the processing mode, cells were harvested, washed three times with PBS, centrifuged and fixed with $70 \%$ anhydrous ethanol overnight at $4{ }^{\circ} \mathrm{C}$. They were then incubated with RNAase for $30 \mathrm{~min}$ and stained with PI for $15 \mathrm{~min}$ at $37^{\circ} \mathrm{C}$ in the dark. The samples were examined by flow cytometry (FACS Calibur, BD, USA), and the data were analyzed with ModFit LT software (FACS Calibur, BD, USA).

\section{Statistical analysis}

The data are expressed as mean as the means \pm SEM. Comparisons were performed using Student's $t$ test or ANOVA as appropriate. $\mathrm{P}<0.05$ was considered significant.
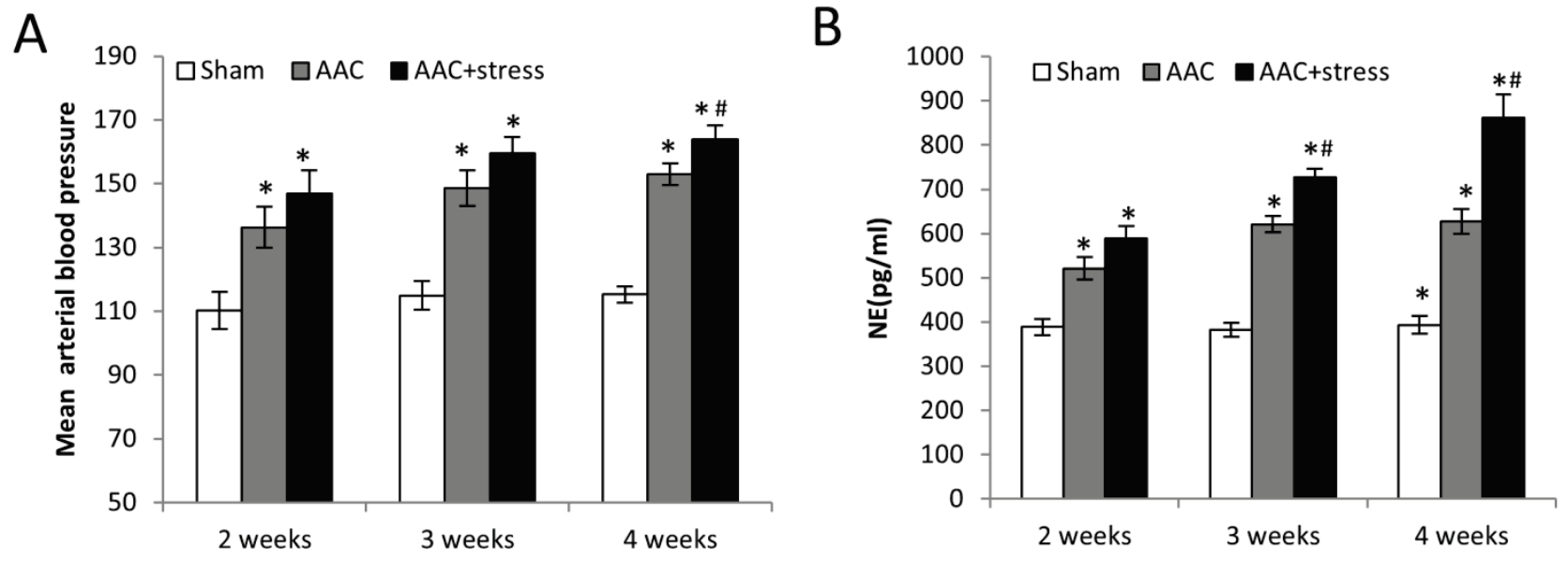

Fig. 1. The levels of blood pressure and NE during the progression of pressure overload. Rats were subjected to abdominal aortic constriction (AAC) surgery or chronic restraint stress. A. The mean arterial blood pressure. B. An ELISA kit (IBL, Germany) was used to detect the NE levels in the serum. Data represent means $\pm S D$. $* P \leq 0.05$ vs. same time sham, ${ }^{\#} P<0.05$ vs. same time $A A C, n=8$.

A

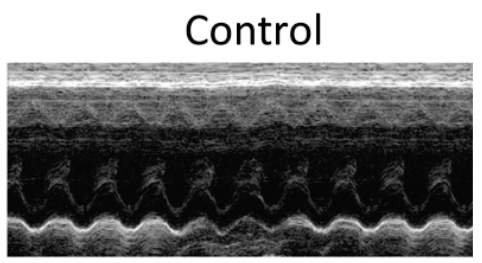

B

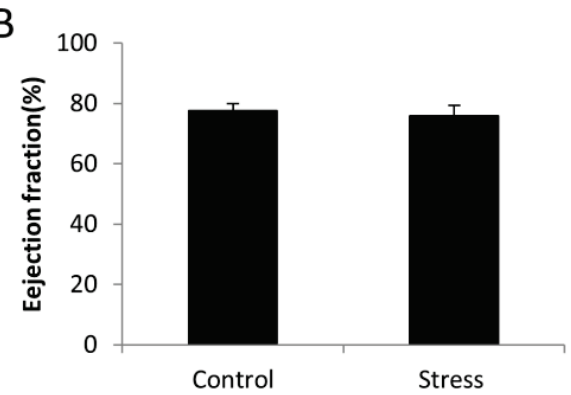

\section{Stress}

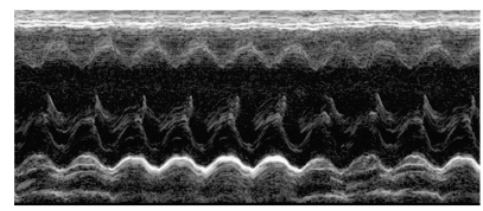

C

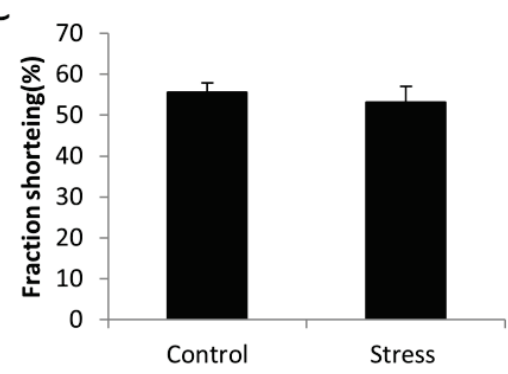

Fig. 2. Echocardiographic parameters of the stress rats. The rats were subjected to chronic restraint stress for $6 \mathrm{~h}$ per day. Echocardiographic assessment were used to detect the cardiac function after 3 weeks of stress. A. Representative left ventricular (LV) M-mode echocardiograms in short axis. B. Left ventricular ejection fraction (\% EF). C. Left ventricular fractional shortening (\% FS). Data represent means $\pm S D$, $\mathrm{n}=8$. 


\section{Results}

Chronic stress worsens pressure overload-induced cardiac dysfunction

To explore the impact of stress on the pressure overload-induced cardiac dysfunction, a microsurgical approach was used to induce hypertension by pressure overload and chronic restraint stress was used after 1 week of AAC operation. Compared with the sham group, the blood pressure of the AAC rats increased after 1 week and remained high for 4 weeks (Fig. 1A). Also, we found that chronic stress induced higher blood pressure than the AAC groups (Fig. 1A). To recognize the cardiac function after the AAC or chronic stress, we used the echocardiographic assessment to detect the cardiac function. Echocardiographic assessment showed no significant decrease of the ejection fraction and fractional shortening induced by 3 weeks stress (Fig. 2). But, compared with the sham group, the left ventricle anterior wall and left ventricular mass increased after 4 weeks of the AAC rats or after 3 weeks of the $\mathrm{AAC}+$ stress rats, indicating that stress promoted pressure overload-induced cardiac hypertrophy (Fig. 3A,B,C). Echocardiographic assessment showed no significant increase of the ejection fraction induced by AAC during our detected times (Fig. 3D). But, the ejection fraction decreased after 3 weeks in the AAC + stress group (Fig. 3D). Also, we found that the fractional shortening significantly decreased after 4 weeks in the AAC + stress group (Fig. 3E). These results illustrated that chronic stress promotes pressure overload-induced cardiac dysfunction.

A

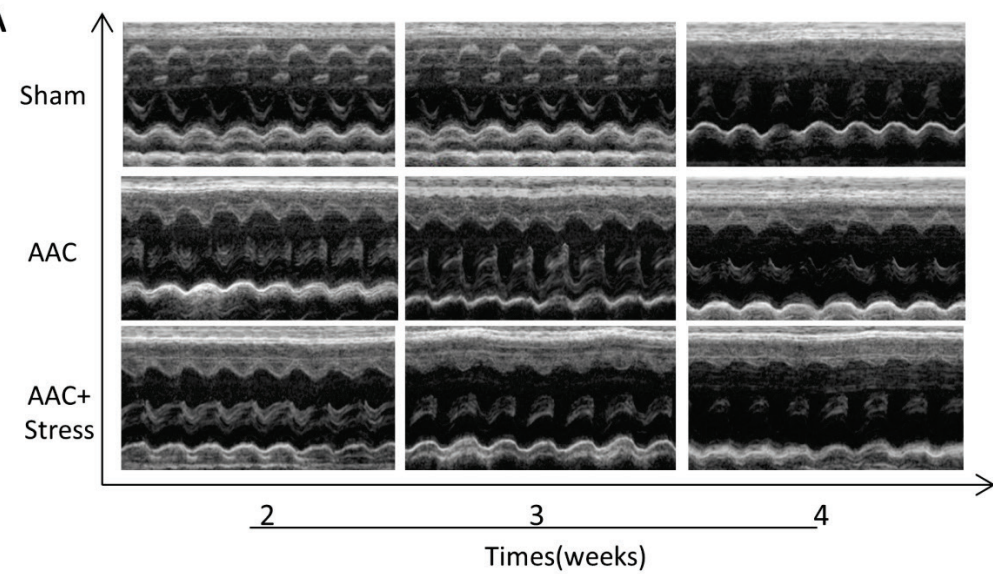

B

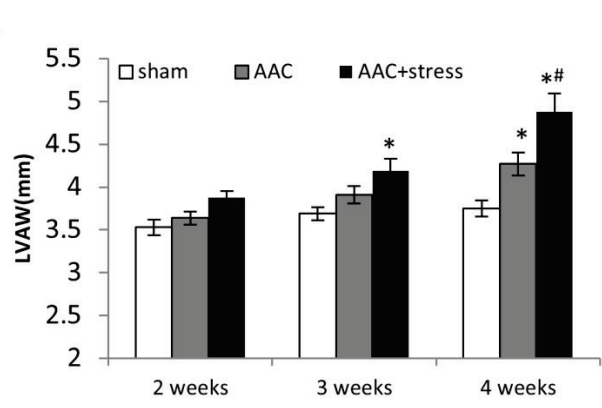

C

D
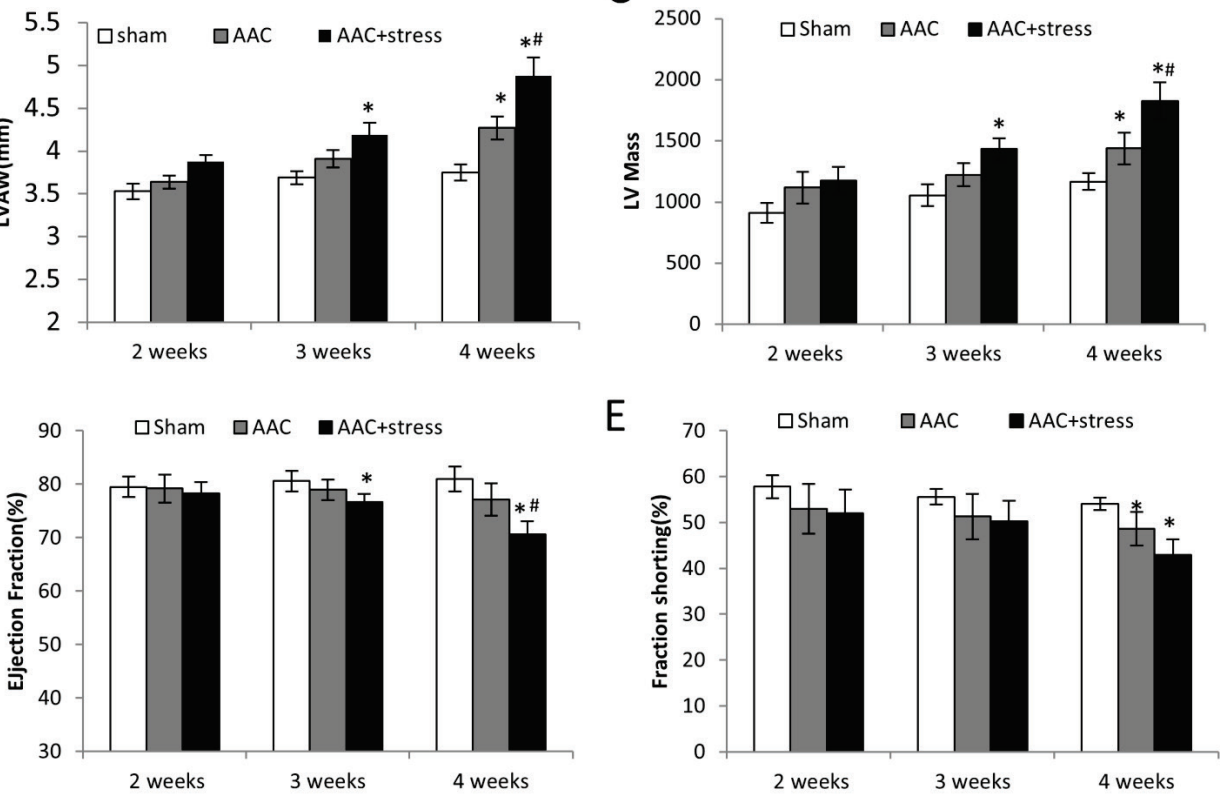

E

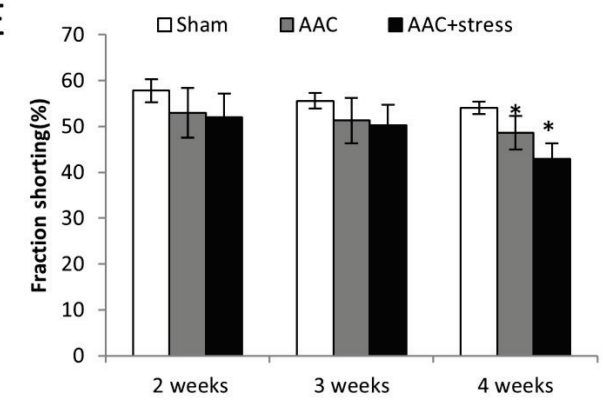

Fig. 3. Echocardiographic parameters of the rats during the progression of pressure overload. Rats were subjected to abdominal aortic constriction (AAC) surgery or chronic restraint stress. A. Representative left ventricular (LV) M-mode echocardiograms in short axis. B. Left ventricular anterior wall (LVAW). C. Left ventricular mass (LV mass). D. Left ventricular ejection fraction (\% EF). E. Left ventricular fractional shortening (\% FS). Data represent means $\pm S D$. ${ }^{*} P \leq 0.05$ vs. same time sham, ${ }^{*} P<0.05$ vs. same time $A A C, n=8$. 

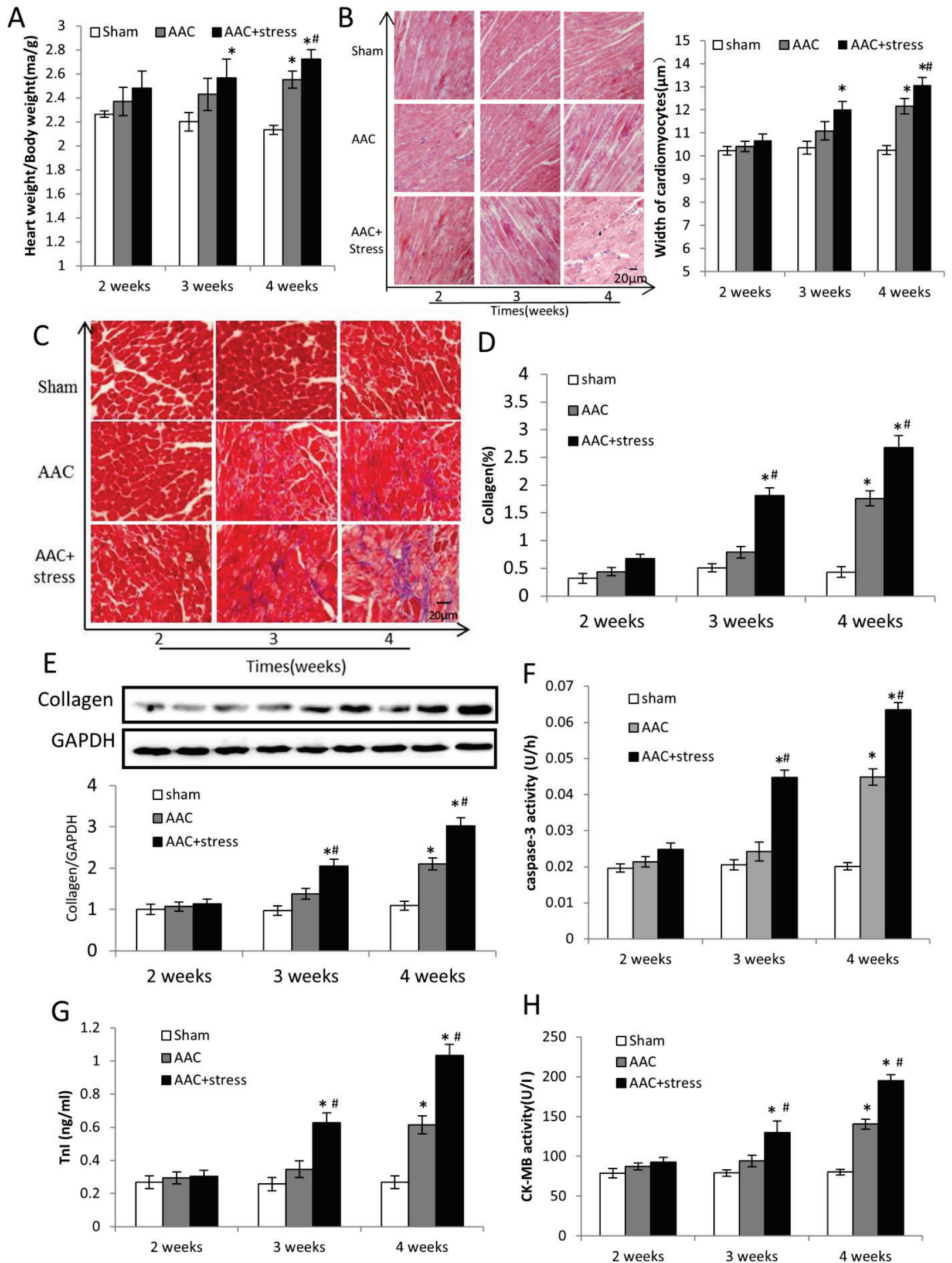

Fig. 4. The biological characteristics of $A A C$ or stress induced myocardial injury. A. Heart weight/body weight ratios. B. Representative histological images with hematoxylin and eosin staining of heart sections. The widths of 30 individual cardiomyocytes were measured across a line bisecting the nucleus, bar graph shows the cardiomyocyte width. C. Representative histological images with Masson's trichrome staining of heart sections. D. Quantification of collagen accumulation areas for Masson's trichrome staining. E. Western blot analysis of collagen expression. F. Caspase-3 activity in myocardial tissues. G. ELISA analysis of the level of CK-MB in the serum. H. ELISA analysis of the level of $\mathrm{TnI}$ in the serum. Data represent means $\pm \mathrm{SD}$. ${ }^{*} \mathrm{P} \leq 0.05$ vs. same time sham, ${ }^{\#} \mathrm{P}<0.05$ vs. same time $A A C, n=8$. 
Pressure overload-induced cardiac hypertrophy and fibrosis can be increased by chronic stress

We detected the change of cardiac hypertrophy after AAC or stress. The heart weight to body weight ratios increased after 4 weeks of the $\mathrm{AAC}$ rats, but increased after 3 weeks of the $\mathrm{AAC}+$ stress rats (Fig. 4A). Echocardiographic assessment demostrated an evident increase in the left ventricle mass and the left ventricle anterior wall after 4 weeks of the AAC rats or after 3 weeks of the $\mathrm{AAC}+$ stress rats (Fig. 3B,C). Hematoxylin and eosin staining demonstrated that the cardiomyocytes width of AAC + stress rat heart sections were significantly thicker than the AAC group (Fig. 4B), indicating that chronic stress promoted pressure overload induced cardiac hypertrophy. Moreover, to determine the effect of stress on pressure overload-induced cardiac fibrosis, we detected the collagen accumulation in the heart sections. Masson staining showed pressure overload-induced collagen accumulation increased when the rats were subjected to chronic restraint stress (Fig. 4C,D). Also, Western blot analysis revealed that the expression of collagen was markedly increased after 4 weeks of the AAC rats or after 3 weeks of the $\mathrm{AAC}+$ stress rats (Fig. 4E). These results demonstrate that pressure overload-induced fibrosis was aggravated by chronic stress.

Chronic stress aggravated pressure overload-induced cardiomyocytes injury

Cardiomyocytes apoptosis in the left ventricular tissue of rat was evaluated by detecting the caspase- 3 activity. Compared to the sham group, there was an increase in LV tissue caspase- 3 activity after 4 weeks of AAC operation. But, the increase of caspase- 3 activity was found as early as 3 weeks in the AAC + stress group (Fig. 4F). Caspase-3 activity in the AAC + stress group was significantly higher than that in the AAC group after 4 weeks of the operation. In order to determine the injury of cardiac myocytes, the amounts of CK-MB and TnI in the serum were detected by ELISA. The content of serum CK-MB and TnI significantly increased in the AAC group after 4 weeks of operation and in the AAC + stress group after 3 weeks of operation (Fig. 4G,H). Moreover, it was significantly higher than in $\mathrm{AAC}$ group after 4 weeks of the operation in $\mathrm{AAC}+$ stress group (Fig. 4G,H). These results demonstrated that chronic stress promoted the progression of pressure overload- induced cardiomyocyte injury.

\section{NE induced cardiomyocytes hypertrophy and apoptosis}

Under the condition of pressure overload or stress, the secretion of norepinephrine (NE) increased. Also, the level of $\mathrm{NE}$ was higher in the $\mathrm{AAC}+$ stress group than that in the AAC group (Fig. 1B). To determine the effect of high concentrations of $\mathrm{NE}$ on cardiomyoctes hypertrophy and apoptosis, the neonatal rat cardiac myocytes were incubated with $10^{-6}-10^{-4} \mathrm{~mol} / 1$ $\mathrm{NE}$ for $48 \mathrm{~h}$. Compared with the parental controls, protein content of cardiac myocytes increased in a dosedependent manner (Fig. 5A). BNP is an important biomarker of hypertrophy, western blotting analysis showed NE had a dose dependent stimulatory effect on BNP expression (Fig. 5B). Furthermore, flow cytometry analysis showed there was a significant increase of cell apoptosis with a dose-dependent manner in the NE-induced cardiomyocytes (Fig. 5C). These results indicated that cardiac myocytes hypertrophy and apoptosis were more obvious in the higher level of NE group, which may be the mechanisms of pressure overload-induced cardiac hypertrophy and injury worsened by chronic stress.

\section{$N E$ induced cardiac fibroblasts proliferation and collagen expression}

To determine the effects of NE on cardiac fibrosis, the neonatal rat cardiac fibroblasts were incubated with $10^{-6}-10^{-4} \mathrm{~mol} / 1 \mathrm{NE}$ for $24 \mathrm{~h}$. Compared with the parental controls, the MTT assay showed a significant increase of the cell proliferation rate in a dose-dependent manner (Fig. 6A). To identify a potential mechanism for NE-induced cell proliferation, the cell cycle distribution was assessed using flow cytometry, which showed that the percentage of cells in the DNA synthesis phase (S phase) significantly increased in a dose-dependent manner (Fig. 6B). Furthermore, collagen, the important extracellular matrix (ECM) component, was also increased in a dosedependent manner in the NE-induced cardiac fibroblasts (Fig. 6C). These results indicated that higher level of NE induced higher proliferation rate and ECM protein expression, which may contribute to the process of that chronic stress promoted pressure overload-induced cardiac fibrosis. 

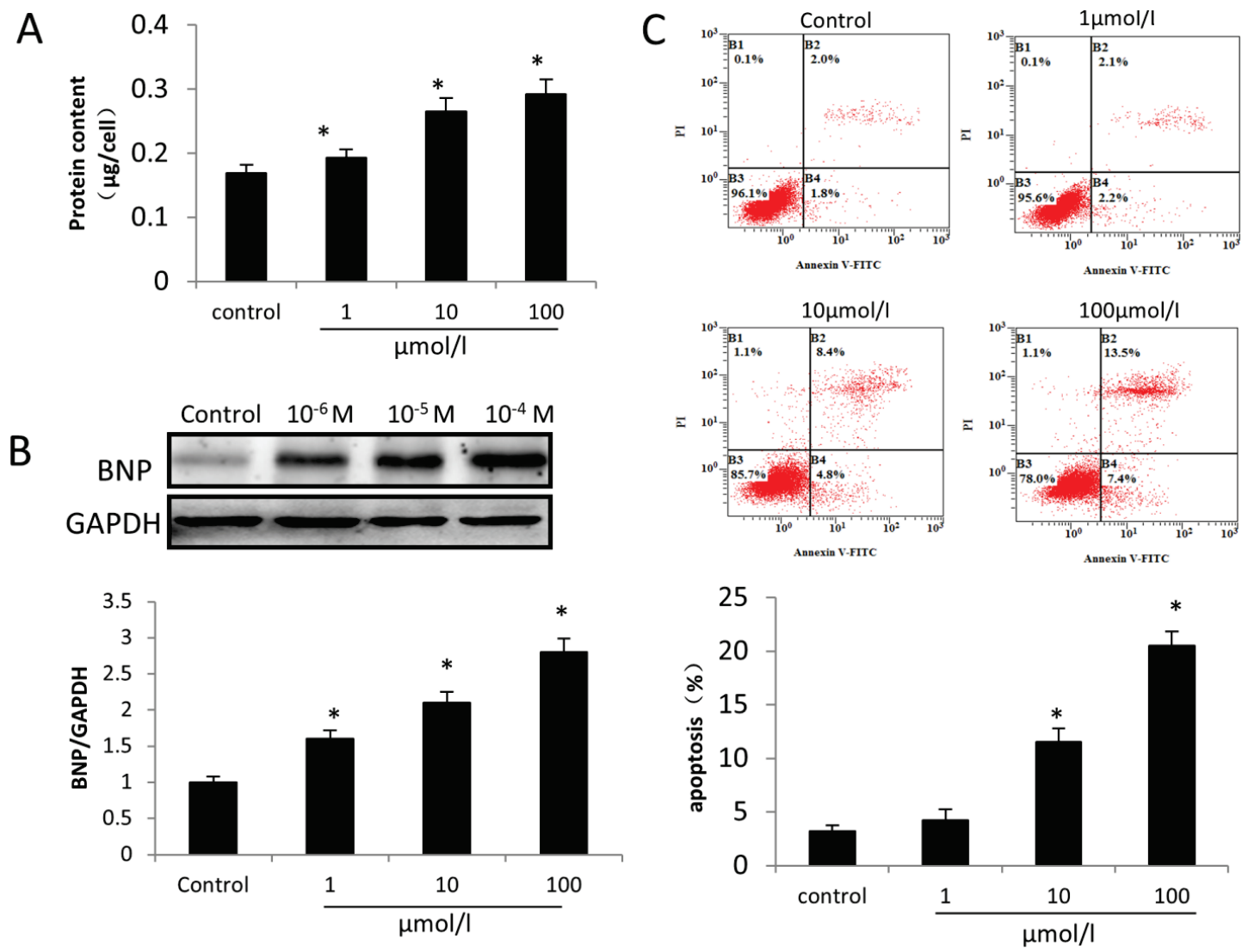

Fig. 5. The effects of NE on cell hypertrophy and apoptosis in cultured cardiac myocytes. Cardiomyocytes were treated with different concentrations of NE for the indicated times. A. The protein content of cell. B. Western blot analysis of NE-induced BNP expression. C. The cells were stained with propidium iodide and annexin $\mathrm{V}$ for flow cytometry and analyzed for apoptosis using CELLQuest software. Data represent means $\pm \mathrm{SD}$. ${ }^{\mathrm{P}} \mathrm{P} \leq 0.05$ vs. Control. All experiments were performed 3 times.

A

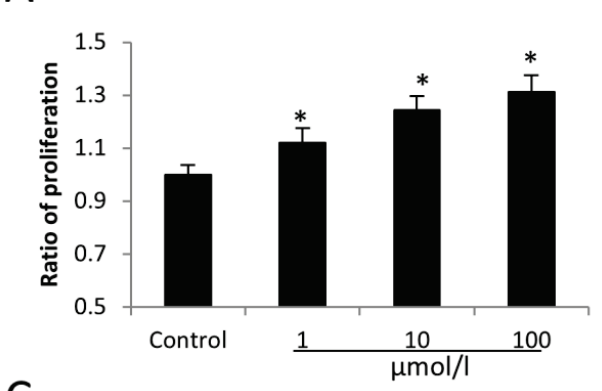

C
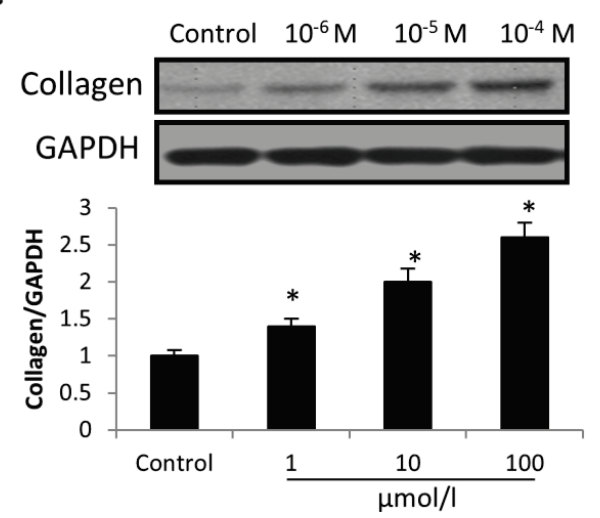

B

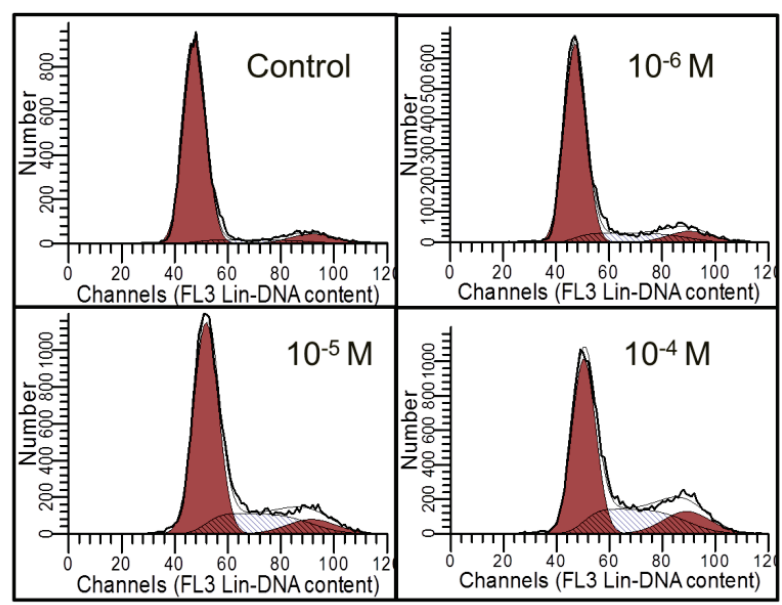

\begin{tabular}{|c|c|c|c|c|}
\hline & Control & $10^{-6} \mathrm{~mol} / /$ & $10^{-5} \mathrm{~mol} / /$ & $10^{-4} \mathrm{~mol} / \mathrm{l}$ \\
\hline \multirow{2}{*}{$\mathrm{G} 1$} & $85.92 \% \pm$ & $77.02 \% \pm$ & $68.13 \% \pm$ & $57.52 \% \pm$ \\
& $2.62 \%$ & $1.52 \%$ & $2.20 \%$ & $2.43 \%$ \\
\hline \multirow{2}{*}{ G2 } & $8.04 \% \pm$ & $8.54 \% \pm$ & $9.32 \% \pm$ & $14.51 \% \pm$ \\
& $0.95 \%$ & $1.06 \%$ & $0.87 \%$ & $1.22 \%$ \\
\hline \multirow{2}{*}{$\mathbf{S}$} & $\mathbf{6 . 0 2} \% \pm$ & $\mathbf{1 4 . 4 7 \% \pm}$ & $\mathbf{2 2 . 5 6} \%$ & $\mathbf{2 8 . 9 6 \% \pm}$ \\
& $\mathbf{0 . 7 3} \%$ & $\mathbf{0 . 9 7 \%}$ & $\mathbf{2 . 7 4 \%}$ & $\mathbf{1 . 9 1 \%}$ \\
\hline
\end{tabular}

Fig. 6. The effects of NE on cell fibrosis in cultured cardiac fibroblasts (CFs). CFs were treated with different concentrations of NE for the indicated times. A. Proliferation was measured using the MTT assay. B. Cells were stained with PI and examined by FACS. The data were analyzed using the ModFit program. C. Western blot analysis of NE-induced collagen expression. Data represent means \pm SD. $* P \leq 0.05$ vs. Control. All experiments were performed 3 times. 


\section{Discussion}

There have been numerous studies about stress, a concept describing the process in which environmental or psychosocial factors exceed the adaptive capacity of an organism, resulting in psychological and biological changes that may place persons at risk for disease (Sparrenberger et al. 2009, Taylor et al. 2010). For instance, stress plays a major role in immunological diseases and immune-related disease processes. In addition, inflammation, infection, autoimmune processes, and perhaps even the onset and development of tumors may occasionally be associated with the stress phenomenon (Esch et al. 2002, Koh et al. 2008, Kashani et al. 2012, Martocchia et al. 2013). Moreover, stress has been considered as an important cause of hypertension among other potential risk factors such as low potassium consumption, low physical activity and sleep abnormalities (Heine and Weiss 1987). Hypertension is one of the most common worldwide diseases. Meanwhile, it is also the most important modifiable risk factor for cardiac remodeling and heart failure. Owing to the associated morbidity and mortality, and the cost to society, hypertension is a serious challenge to public health. Concerted effort on the part of the health care professionals has led to decreased morbidity and mortality from the multiple organ damage arising from long-term and untreated hypertension (Ettner et al. 2012). Stress, as a risk factor for hypertension, should be highly concern. Our research showed that chronic stress increased the AAC-induced high blood pressure and made the pressure overload-induced cardiac dysfunction worsened.

Cardiac remodeling after long-term pressure overload results in ventricular dysfunction and heart failure and is considered to be a key determinant of clinical outcome in heart disease (Levy et al. 2002, Kehat 2012). Cardiac remodeling includes changes in heart size, shape and function in response to injury or stress stimulation. Pathological remodeling involves the reactivation of cardiomyocytes hypertrophy and death and cardiac fibrosis (Kudo et al. 2007, Saito et al. 2010). Our results demonstrated that chronic stress aggravated pressure overload-induced cardiac hypertrophy, cardiac fibrosis and cardiomyocytes apoptosis, which may contribute to the decline of cardiac function. Loss of cardiomyocytes through apoptosis is an important pathological change in myocardial injury (Fan et al. 2013). When the structure of cardiomyocytes damaged, amounts of CK-MB, $\mathrm{LDH}$ and TnI were released into blood, so the content of TnI and CK-MB level was usually used to identify the cell injury and membrane integrity in previous studies (Li et al. 2012, Amani et al. 2013). Our results showed that the contents of these enzymes in AAC + stress rat serum increased significantly, indicating that pressure overload-induced myocardial injury can be aggravated by chronic stress.

The impact of stress on the development of hypertension is believed to involve a sympathetic nervous system response, in which the release of catecholamines leads to increased heart rate, cardiac output, and BP (Carroll et al. 2005, Spruill 2010). As shown in Figure 1B, compared with the AAC group, the level of $\mathrm{NE}$ is higher in the $\mathrm{AAC}+$ stress group. The heart is comprised of a syncytium of cardiac myocytes and surrounding nonmyocytes, the majority of which are cardiac fibroblasts (Zhang et al. 2012). Cardiomyocyte hypertrophy and apoptosis, cardiac fibroblasts proliferation and ECM protein synthesis are considered to be the important cellular basis for pressure overloadinduced cardiac remodeling (Wollert and Drexler 2002, Fan et al. 2012). As is well known, NE binds to specific adrenoceptor on the cell membrane to induce PKC activation and cause a variety of cellular effects. We are currently performing further investigations about the effect of high level of NE induced by chronic stress on the cardiac myocytes and fibroblasts. Compared with the parental controls, NE had a significantly stimulatory effect on cardiomyocytes hypertrophy and apoptosis, cardiac fibroblasts proliferation and ECM protein synthesis in a dose-dependent manner, which may contribute to the high level of NE induced by chronic stress to promote pressure overload-induced cardiac dysfunction.

In summary, we demonstrated that chronic stress promotes the progression of pressure overload-induced cardiac dysfunction. We further verified that pressure overload-induced myocardial hypertrophy, cardiac fibrosis, cardiomyocyte injury and apoptosis could be aggravated by chronic stress. The high level of NE induced by chronic stress contributed to acceleration of pressure overload-induced cardiac dysfunction through inducing cardiac myocytes hypertrophy and apoptosis, cardiac fibroblasts proliferation and collagen synthesis. These findings improved our understanding of the risk factors and mechanisms involved in the effect of stress on pressure overload-induced cardiac dysfunction and provided new insights into future therapeutic targets for 
hypertension-induced cardiac remodeling and heart failure.

\section{Conflict of Interest}

There is no conflict of interest.

\section{Acknowledgements}

This research was supported by the Chinese National Natural Science Foundation (grant no. 31071022).

\section{References}

AMANI M, JEDDI S, AHMADIASL N, USEFZADE N, ZAMAN J: Effect of HEMADO on level of CK-MB and LDH enzymes after ischemia/reperfusion injury in isolated rat heart. Bioimpacts 3: 101-104, 2013.

BRILLA CG, PICK R, TAN LB, JANICKI JS, WEBER KT: Remodeling of the rat right and left ventricles in experimental hypertension. Circ Res 67: 1355-1364, 1990.

BROWN RD, AMBLER SK, MITCHELL MD, LONG CS: The cardiac fibroblast: therapeutic target in myocardial remodeling and failure. Annu Rev Pharmacol Toxicol 45: 657-687, 2005.

CARROLL D, PHILLIPS AC, RING C, DER G, HUNT K: Life events and hemodynamic stress reactivity in the middle-aged and elderly. Psychophysiology 42: 269-276, 2005.

CUMBERMACK KM, CHENG J, NONG Y, MAHLE WT, JOYNER RW, BORDER WL, WAGNER MB, FYFE DA, LEONG T, WANG Y: A juvenile murine heart failure model of pressure overload. Pediatr Cardiol 32: 145-153, 2011.

ESCH T, STEFANO GB, FRICCHIONE GL, BENSON H: Stress-related diseases - a potential role for nitric oxide. Med Sci Monit 8: RA103- RA118, 2002.

ETTNER R, ETTNER F, WHITE T: Secrecy and the pathogenesis of hypertension. Int J Family Med 2012: 492718, 2012.

FAN D, TAKAWALE A, LEE J, KASSIRI Z: Cardiac fibroblasts, fibrosis and extracellular matrix remodeling in heart disease. Fibrogenesis Tissue Repair 5: 15, 2012.

FAN Q, HUANG ZM, BOUCHER M, SHANG X, ZUO L, BRINKS H, LAU WB, ZHANG J, CHUPRUN JK, GAO E: Inhibition of Fas-associated death domain-containing protein (FADD) protects against myocardial ischemia/reperfusion injury in a heart failure mouse model. PLoS One 8: e73537, 2013.

HEINE H, WEISS M: Life stress and hypertension. Eur Heart J 8 (Suppl B): 45-55, 1987.

IPPOLITI F, CANITANO N, BUSINARO R: Stress and obesity as risk factors in cardiovascular diseases: a neuroimmune perspective. J Neuroimmune Pharmacol 8: 212-226, 2013.

JIN H, LIU AD, HOLMBERG L, ZHAO M, CHEN S, YANG J, SUN Y, TANG C, DU J: The role of sulfur dioxide in the regulation of mitochondrion-related cardiomyocyte apoptosis in rats with isopropylarterenol-induced myocardial injury. Int J Mol Sci 14: 10465-10482, 2013.

KASHANI F, BABAEE S, BAHRAMI M, VALIANI M: The effects of relaxation on reducing depression, anxiety and stress in women who underwent mastectomy for breast cancer. Iran J Nurs Midwifery Res 17: 30-33, 2012.

KEHAT I: Novel strategies for the treatment of heart failure. Rambam Maimonides Med J 3: e0011, 2012.

KOH KB, LEE Y, BEYN KM, CHU SH, KIM DM: Counter-stress effects of relaxation on proinflammatory and antiinflammatory cytokines. Brain Behav Immun 22: 1130-1137, 2008.

KREMNEVA LV, ABATUROVA OV: Molecular and cellular mechanisms of myocardial remodeling in heart failure (in Russian). Klin Med (Mosk) 81: 4-7, 2003.

KUDO H, KAI H, IMAIZUMI T: Cardiac remodeling (in Japanese). Nihon Rinsho 65 (Suppl 4): 302-306, 2007.

LEVY D, KENCHAIAH S, LARSON MG, BENJAMIN EJ, KUPKA MJ, HO KK, MURABITO JM, VASAN RS: Long-term trends in the incidence of and survival with heart failure. $N$ Engl J Med 347: 1397-1402, 2002.

LI H, XIE YH, YANG Q, WANG SW, ZHANG BL, WANG JB, CAO W, BI LL, SUN JY, MIAO S, HU J, ZHOU XX, QIU PC: Cardioprotective effect of paeonol and danshensu combination on isoproterenol-induced myocardial injury in rats. PLoS One 7: e48872, 2012.

LI J, LEVICK SP, DIPETTE DJ, JANICKI JS, SUPOWIT SC: Alpha-calcitonin gene-related peptide is protective against pressure overload-induced heart failure. Regul Pept 185: 20-28, 2013. 
LUTGENDORF S, LOGAN H, KIRCHNER HL, ROTHROCK N, SVENGALIS S, IVERSON K, LUBAROFF D: Effects of relaxation and stress on the capsaicin-induced local inflammatory response. Psychosom Med 62: 524-534, 2000.

MANOLIS AJ, POULIMENOS LE, KALLISTRATOS MS, GAVRAS I, GAVRAS H: Sympathetic overactivity in hypertension and cardiovascular disease. Curr Vasc Pharmacol 12: 4-15, 2014.

MARTOCCHIA A, STEFANELLI M, FALASCHI GM, TOUSSAN L, ROCCHIETTI MARCH M, RAJA S, ROMANO G, FALASCHI P: Targets of anti-glucocorticoid therapy for stress-related diseases. Recent Pat CNS Drug Discov 8: 79-87, 2013.

RIBEIRO RF JR, DABKOWSKI ER, O'CONNELL KA, XU W, GALVAO T DE F, HECKER PA, SHEKAR KC, STEFANON I, STANLEY WC: Effect of a high-protein diet on development of heart failure in response to pressure overload. Appl Physiol Nutr Metab 39: 238-247, 2014.

SAITO S, MATSUMIYA G, SAKAGUCHI T, MIYAGAWA S, YAMAUCHI T, KURATANI T, SAWA Y: Cardiac fibrosis and cellular hypertrophy decrease the degree of reverse remodeling and improvement in cardiac function during left ventricular assist. J Heart Lung Transplant 29: 672-679, 2010.

SPARRENBERGER F, CICHELERO FT, ASCOLI AM, FONSECA FP, WEISS G, BERWANGER O, FUCHS SC, MOREIRA LB, FUCHS FD: Does psychosocial stress cause hypertension? A systematic review of observational studies. J Hum Hypertens 23: 12-19, 2009.

SPRUILL TM: Chronic psychosocial stress and hypertension. Curr Hypertens Rep 12: 10-16, 2010.

TAYLOR M, BARR M, STEVENS G, BRYSON-TAYLOR D, AGHO K, JACOBS J, RAPHAEL B: Psychosocial stress and strategies for managing adversity: measuring population resilience in New South Wales, Australia. Popul Health Metr 8: 28, 2010.

TSURUDA T, KATO J, KITAMURA K, KAWAMOTO M, KUWASAKO K, IMAMURA T, KOIWAYA Y, TSUJI T, KANGAWA K, ETO T: An autocrine or a paracrine role of adrenomedullin in modulating cardiac fibroblast growth. Cardiovasc Res 43: 958-967, 1999.

WANG S, SONG P, ZOU MH: AMP-activated protein kinase, stress responses and cardiovascular diseases. Clin Sci (Lond) 122: 555-573, 2012.

WENNEBERG SR, SCHNEIDER RH, WALTON KG, MACLEAN CR, LEVITSKY DK, SALERNO JW, WALLACE RK, MANDARINO JV, RAINFORTH MV, WAZIRI R: A controlled study of the effects of the Transcendental Meditation program on cardiovascular reactivity and ambulatory blood pressure. Int $J$ Neurosci 89: 15-28, 1997.

WOLLERT KC, DREXLER H: Regulation of cardiac remodeling by nitric oxide: focus on cardiac myocyte hypertrophy and apoptosis. Heart Fail Rev 7: 317-325, 2002.

YU Y, ZHANG S, WANG G, HONG X, MALLOW EB, WALKER SO, PEARSON C, HEFFNER L, ZUCKERMAN $\mathrm{B}$, WANG X: The combined association of psychosocial stress and chronic hypertension with preeclampsia. Am J Obstet Gynecol 209: 438 e1-438 e12, 2013.

ZHANG P, SU J, MENDE U: Cross talk between cardiac myocytes and fibroblasts: from multiscale investigative approaches to mechanisms and functional consequences. Am J Physiol Heart Circ Physiol 303: H1385-H1396, 2012.

ZHAO Y, WANG W, QIAN L: Hsp70 may protect cardiomyocytes from stress-induced injury by inhibiting Fas-mediated apoptosis. Cell Stress Chaperones 12: 83-95, 2007. 